\title{
MODELISATION ET IDENTIFICATION \\ D'UNE RELATION PLUIE-DEBIT
}

Le modèze "SEMOTS"

\author{
Bernard LORENT \\ Centre Interuniversitaire \\ des Sciences de 1'Environnement \\ Fondation Universitaire Luxembourgeoise \\ rue des Déportés, 140 \\ 6700 - ARLON (Belgique)
}

RESUME : $\quad$ Cet article présente un modèle performant de prévision et de simulation du débit d'une rivière à 1 'exutoire de son bassin versant. Ce modèle est construit à partir de la connaissance du débit à 1 'exutoire, des précipitations en plusieurs stations ainsi que d'une estimation de l'évapotranspiration potentielle. Un premier sous-modèle nonlinéaire, permet le calcul d'une pluie nette, d'une fonction de stockage superficiel et d'un terme d'infiltration. Ensuite, un second sous-modèle simple simule le débit de base de la rivière. Enfin, un modèle utilisant une équation aux différences, décrit le processus pluie nette-débit de ruissellement. A la fin de cet article, les résultats pratiquement obtenus sont présentês et discutés.

\section{INTRODUCTION.}

I.1. Nous présentons dans ce texte, quelques résultats importants d'une étude en cours, dont l'objectif principal est la réalisation d'un modèle de prévision et de simulation des débits de la Semois à l'exutoire de son bassin versant belge (Membre). Pour atteindre cet objectif, nous utilisons actuellement des mesures journalières collectées de 1967 à 1973. Ces données sont : le débit de la rivière à Membre, une estimation de l'évapotranspiration potentielle du bassin versant [1] et des relevés pluviométriques en quelques dix stations. Ces données nous ont été aimablement fournies par les soins de la section d'Hydrologie de l'Institut Royal Météorologique (I.R.M.) et du Service d'Etude Hydrologique (SETHY) du Ministère des Travaux Publics.

Précisons enfin que la Semois est un affluent ardennais de la Meuse et que son bassin versant limité à Membre a une superficie de $1229 \mathrm{~km}^{2}$. Le lecteur désireux d'avoir une description détaillée des caractêristiques hydrométéorologiques de ce bassin lira avec intêrêt la référence [2].

I.2. S'il nous fallait concevoir un modẽle de relation pluie-débit à partir de 1 'observation des phénomènes physiques qui interviennent dans ce processus, notre tâche deviendrait rapidement impossible. En effet, en tout lieu, il conviendrait de 
préciser les caractères physiques de sol, de pente, de couverture végétale ... et ensuite de décrire l'iriteraction entre ces différents éléments et les conditions météorologiques pour tenter d'estimer localement l'infiltration, le stockage superficie1, l'écoulement de surface ... Et après avoir mis en équation tous les phénomènes complexes localement déterminants, il faudrait encore préciser comment toute cette eau parvient à l'exutoire....

Fort heureusement, une alternative s'offre à nous. En effet, la dynamique de la relation pluie-débit est directement fonction des caractères physiques du bassin. Nous allons dès lors considêrer le bassin comne une boite noire dont nous étudierons uniquement les caractéristiques entrées-sorties.

La relation pluie-évaporation-débit est toutefois non-linéaire et ses caractéristiques varient suivant la saison. Aussi, reviendrons-nous à certaines considêrations hydrologiques importantes afin de dégager une structure de modèle simple et proche de la réalité physique. Nous sommes bien sûr conscients de ce que nous ferons de considérables simplifications dans la description des phénomènes ma is nous le ferons, convâincus de ce que cette méthode conduit à des modēles de prêvision et de simulation efficaces.

I.3. Le modèle présenté est conçu en temps discret. La période d'échantillonnage sera désignée par $T$ et nous ne considèrerons donc les différentes grandeurs qu'aux instants : $(k-1) \cdot T, k . T,(k+1) . T \ldots$ $X(k)$ représentera a insi la grandeur $X$ au temps $k . T$

Toutefois, en raison du type de mesuresutilisées, les grandeurs $X(k)$ représenteront le plus souvent non pas des valeurs instantanées mais des moyennes sur l'intervalle de temps $k$ compris entre $(k-1 / 2)$. T et $(k+1 / 2) . T$, c'est-à-dire que :

$$
x(k)=\frac{1}{T} \int_{(k-1 / 2) \cdot T}^{(k+1 / 2) \cdot T} x(t) \cdot d t
$$

II. DESCRIPTION GLOBALE DU MODELE $[3],[4],[5]$.

II.1. Appelons $Q(k)$ le débit de la rivière à l'instant $k$. Nous considérons, de manière classique, que ce débit est composé de deux termes, l'un le débit de ruissellement $R(k)$ et 7 'autre le débit de base $B(k)$.

Le débit de ruissellement $R(k)$ sera la fraction du débit que nous supposerons ètre due directement aux pluies du fait du ruissellement superficiel.

Par contre, le débit de base $B(k)$ est un terme que nous supposerons provenir de 1a vidange des nappes et qui dépend des pluies moyennant un terme d'infiltration en profondeur. 
Nous écrirons donc:

$$
Q(k)=B(k)+R(k)
$$

Lorsque le terme $R(k)$ devient négligeable, nous dirons que nous sommes en pêriode d'étiage. Par contre, lorsque le terme $R(k)$ est important, nous dirons que nous sommes en période de crue.

II.2. Le volume total d'eau $\mathrm{PB}(k)$ précipitéen un point durant l'intervalle de temps $k$ peut se décomposer comme suit :

$$
P B(k)=P N(k)+E 1(k)+S I(k)
$$

où : PN(k) est la pluie nette, c'est-à-dire la fraction de la pluie $P B(k)$ qui est supposée atteindre 1'exutoire du bassin par ruissellement superficiel et qui influencera rapidement le débit de la rivière. La pluie nette $\mathrm{PN}(k)$ engendrera le débit de ruissellement $R(k)$.

$E 1(k)$ est la fraction du volume d'eau $\mathrm{PB}(k)$ qui sera évaporée durant l'intervalle de temps $k$.

$\mathrm{SI}(k)$ est la fraction de la pluie $\mathrm{PB}(\mathrm{k})$ qui est supposée rester sur place à la fin de 1'intervalle de temps $k$, soit sur la vēgétation par interception, soit dans des dépressions superficielles ou dans les quelques premiers centimètres du sol. Une partie de ce volume $S I(k)$ s'infiltrera ensuite en profondeur tandis qu'une autre s'évaporera plus tard, soit directement, soit ã travers les mécanismes de transpiration des plantes.

Le coefficient de ruissellement au temps $k$ est défini comme suit :

$$
r(k)=\frac{P N(k)}{P B(k)}
$$

II.3. A la surface du sol, nous écrirons l'équation de continuité :

$$
S(k)=S(k-1)+S I(k)-I(k)-E 2(k)
$$

où : $S(k)$, que nous appellerons le "stockage superficiel ", représente le volume d'eau accumulé à la surface du sol à la fin de l'intervalle de temps $k$, cette accumulation se faisant, soit dans la végëtation, soit dans les dépressions superficielles ou encore dans les quelques premiers centimètres du sol. 
$I(k)$ est le volume d'eau qui s'infiltre en profondeur vers les nappes durant $l$ 'intervalla de temps $k$.

E2(k) est le volume d'eau qui s'évapore à partir du stockage superficiel, soit directement, soit par transpiration.

11.4. Le terme d'infiltration $I(k)$ alimente les nappes qui fournissent Te débit de base $B(k)$. Nous considèrerons donc $B(k)$ comme une fonction de $I(k)$.

II.5. Le processus piuie-débit est finalement décrit par le schéma de

la figure 1.

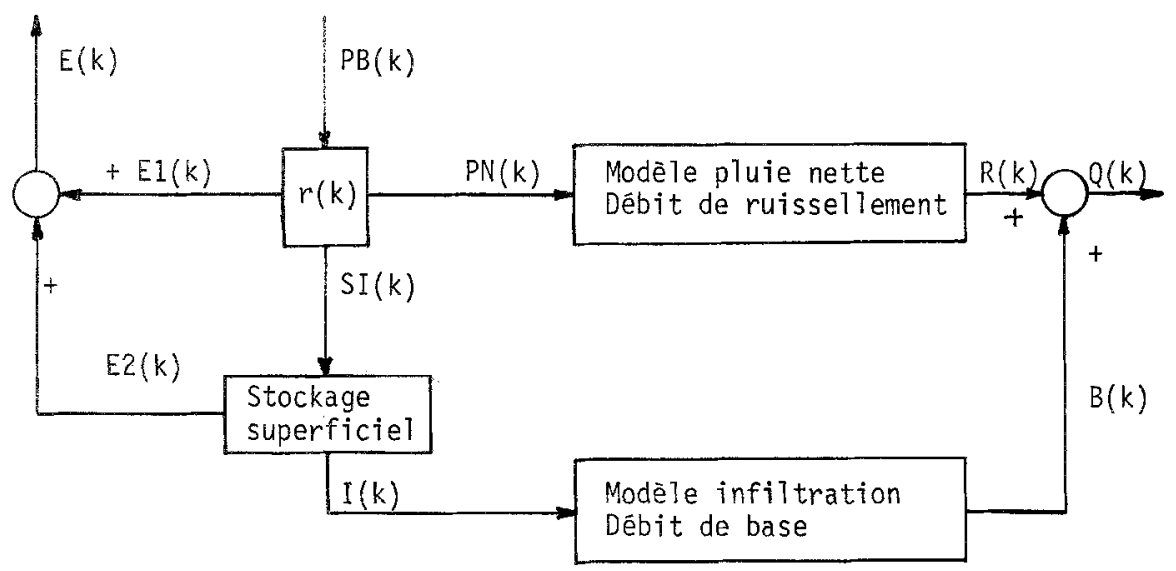

Fig. 1

On observera que 1 'évaporation totale résulte de l'addition des termes $\mathrm{E} 1(k)$ et $\mathrm{E} 2(k)$.

Remarque : Idéalement, un terme correspondant ã la remontée capillaire des eaux de nappes vers la surface devrait être introduit : ceci peut être effectué par exemple en admettant que $I(k)$ peut prendre des valeurs négatives.

\section{ii. Le MODELE de CALCUL DE LA PLUIE NeTte $[6],[7]$.}

III.I. S'il est relativement aisê de schêmatiser les phénomènes par les ëquations de bilan (2) et (4), il est beaucoup plus difficile, sinon parfois impossible, de quantifier rigoureusement les lois physiques qui régissent ces relations. Localement, le problème est déjã bien ardu, et $i 1$ devient encore plus complexe à 1 'échelle d'un bassin en raison de la diversité des caractéristiques dans l'espace. Aussi, afin de surmonter cette difficulté, proposons-nous un modèle non-linéaire 
basē sur les quelques importantes observations hydrologiques suivantes :

- Le coefficient de ruissellement augmente avec la saturation du sol en eau : un sol et une végétation sèche pourront absorber beaucoup d'eau et par contre, une végétation détrempée et un sol saturé d'eau provoqueront un ruissellement important. Dès lors, nous lierons le coefficient de ruissellement au stockage superficiel en sorte que pour un stockage faible, le coefficient de ruissellement soit proche de zêro et que pour un stockage élevé, le coefficient de ruissellement soit proche de l'unité.

- Pour un état donné de l'humidité du sol et de la végétation au début d'une averse, le coefficient de ruissellement augmente avec l'intensité de l'averse : ceci signifie que, dans notre modèle, le stockage superficiel $\mathrm{S}(\mathrm{k}-1)$ au début de l'intervalle de temps $k$ ne déterminera pas un coefficient de ruissellement unique mais un ensemble de coefficients de ruissel Tement qui seront fonction du volume d'eau $P B(k)$ précipitée durant 1 'intervalle de temps $k$.

- L'infiltration est importante lorsque le sol est saturé en eau et elle devient nulle quand le sol est sec ou faiblement humide : notre modèle devra également en tenir compte.

III.2. Ces observations nous conduisent ainsi au modèle dēcrit ci-dessous : III.2.1. Considérons les deux relations déjà proposêes :

$$
\begin{aligned}
& P B(k)=P N(k)+E I(k)+S I(k) \\
& S(k)=S(k-1)+S I(k)-I(k)-E 2(k)
\end{aligned}
$$

Ces deux relations peuvent être considérées en des endroits précis, ou bien à l'échelle d'un bassin ou d'un sous-bassin.

Supposons que le stockage superficiel $S(k)$ ait une valeur maximale que nous appellerons $\underline{S m a x}$. Cette valeur maximale de $S(k)$ correspond à un sol tout à fait saturé en eau et à une végétation totalement détrempée. Introduisons maintenant la notion de déficit de stockage en désignant ce déficit par la différence entre Smax et $S(k)$. On écrira :

$$
D(k)=S \max -S(k)
$$

Le rapport $S(k) / S m a x$ devient dans notre modẽle un indice de saturation en eau du bassin tandis que le rapport $D(k) / S m a x$ correspond à un indice de capacjté d'absorption de 1'eau de pluie. 
III.2.2. Appelons ETP(k) l'évapotranspiration potentielle durant l'intervalle de temps $k$ et supposons, pour plus de simplicité, que l'évaporation se fait à un taux potentiel d'abord à partir de 1 'eau précipitée durant 1 'intervalle de temps considéré. Nous distinguerons deux situations :

a) $P B(k) \leqslant E T P(k)$

c'est-à-dire que l'êvapotranspiration potentielle excède le volume d'eau précipitée. Dans ce cas, nous considèrons que toute l'eau précipitée est directement évaporée et qu'il n'y a ni ruissellement,ni eau stockée sur place à 1 'issue de l'intervalle de temps considéré. Dès lors: $E 1(k)=P B(k)$, $\operatorname{PN}(k)=0, S I(k)=0$

b) $\mathrm{PB}(k)>\mathrm{ETP}(k)$

Dans ce cas-ci, il y aura possibilité de ruissellement et de stockage.

Comme nous supposons, comme précisé plus haut, que 1 'évaporation se

fait au taux potentiel d'abord à partir de l'eau précipitée, nous avons d'abord: $E 1(k)=\operatorname{ETP}(k)$

Désignons par PE(k) le volume d'eau qui reste disponible pour le ruissellement et le stockage superficiel : $P E(k)=P B(k)-E T P(k)$

Il nous faut déterminer maintenant une 70 i de répartition entre les termes $S I(k)$ et $P N(k)$.

En raison des phénomènes physiques évoqués plus haut, nous proposons une 10 i de répartition du type :

$$
S I(k)=D(k-1) \cdot\left(1-e^{-P E(k) / b}\right)
$$

avec la condition : $b \geqslant D(k-1)$ qui est imposée du fait que $S I(k)$ doit toujours être infërieur à $P E(k)$. Dès lors :

$$
\begin{aligned}
P N(k) & =P E(k)-S I(k) \\
\text { ou } P N(k) & =P B(k)-E T P(k)-D(k-1) \cdot\left(1-e^{-(P B(k)-E T P(k)) / b}\right)
\end{aligned}
$$

Les relations $S I=f(D, P E)$ et $P N=f(D, P E)$ se trouvent clairement $i 1-$ lustrées par les graphiques des figures 2 et 3 pour diffërentes valeurs de $D(k-1)$.

III.2.3. Nous avons ainsi déterminé les relations qui commandent l'équation de bilan (2).

Pour l'équation de bilan (4), considêrons à nouveau les deux situations

a) $P B(k) \leqslant E T P(k)$

Nous avons ãlors également $\operatorname{El}(k) \leqslant E T P(k)$. Nous considérons qu'il peut alors $y$ 
avoir évaporation à partir du stockage superficiel, et qu'elle se fera en sorte que 1 'évapotranspiration totale atteigne le taux potentiel à condition que le stockage superficiel soit suffisant. Dès lors, si : $S(k-1) \geqslant E T P(k)-E 1(k)$ on prendra : $E 2(k)=\operatorname{ETP}(k)-E 1(k)$

$$
\text { Par contre, si: } S(k-1)<E T P(k)-E 1(k) \text { alors: } E 2(k)=S(k-1)
$$

b) $\mathrm{PB}(k)>\mathrm{ETP}(k)$

Dans ce cas, nous considèrerons que toute l'évaporation s'est opérée à partir de $P B(k)$ et nous aurons donc: $E 2(k)=0$

Finalement, en ce qui concerne le terme d'infiltration en profondeur $I(k)$, il est plausible, à défaut de mieux, de supposer qu'il est proportionnel à un taux de saturation en eau de la surface du lieu considêré et d'écrire :

$$
I(k)=I_{\max } \cdot \frac{S(k-1)+S I(k)-E 2(k)}{S \max }
$$

et, en posant $A=I \max / S \max$, on a finalement :

$$
S(k)=(1-A) \cdot(S(k-1)+S I(k)-E 2(k))
$$

On observera qu'ainsi $S(k) n$ 'excèdera jamais la valeur de (1-A). Smax Il y aura donc toujours une fraction de $P B(k)$ qui sera absorbée pour compenser l'infiltration.

Le schéma de la figure 4 résume le fonctionnement du modèle.

III.3. Actuellement, les valeurs des paramètres que nous avons retenues pour le calcul de la pluie nette journalière moyenne dans le bassin de la Semois sont :

$$
\begin{aligned}
\text { Smax } & =85 \mathrm{~mm} \text { de hauteur d'eau } \\
I \max & =1 \mathrm{~mm} / \text { jour } \\
\mathrm{b} \quad & =\mathrm{D}(\mathrm{k}-1) / 0.875
\end{aligned}
$$

Ces valeurs sont choisies par minimisation de l'erreur quadratique moyenne du modèle global tout en tenant compte d'une conservation des volumes d'eau entre la pluie nette et le débit de ruissellement.

La figure 5 illustre clairement l'efficacité de la méthode appliquée au bassin de la Semois. On observera de haut en bas, les courbes : de T'évapotranspiration potentielle (ETP) - de la pluie brute moyenne (PB) - du stockage superficiel (S) - de la pluie nette (PN) - du débit à Membre (Q). Les unitēs utilisées sont le $0.1 \mathrm{~mm} /$ jour ou le $0.1 \mathrm{~mm}$. Le débit a donc été ramené à sa lame d'eau équivalente. Les performances de ce modèle sont quant à elles explicitées au paragraphe VI de cet article. 

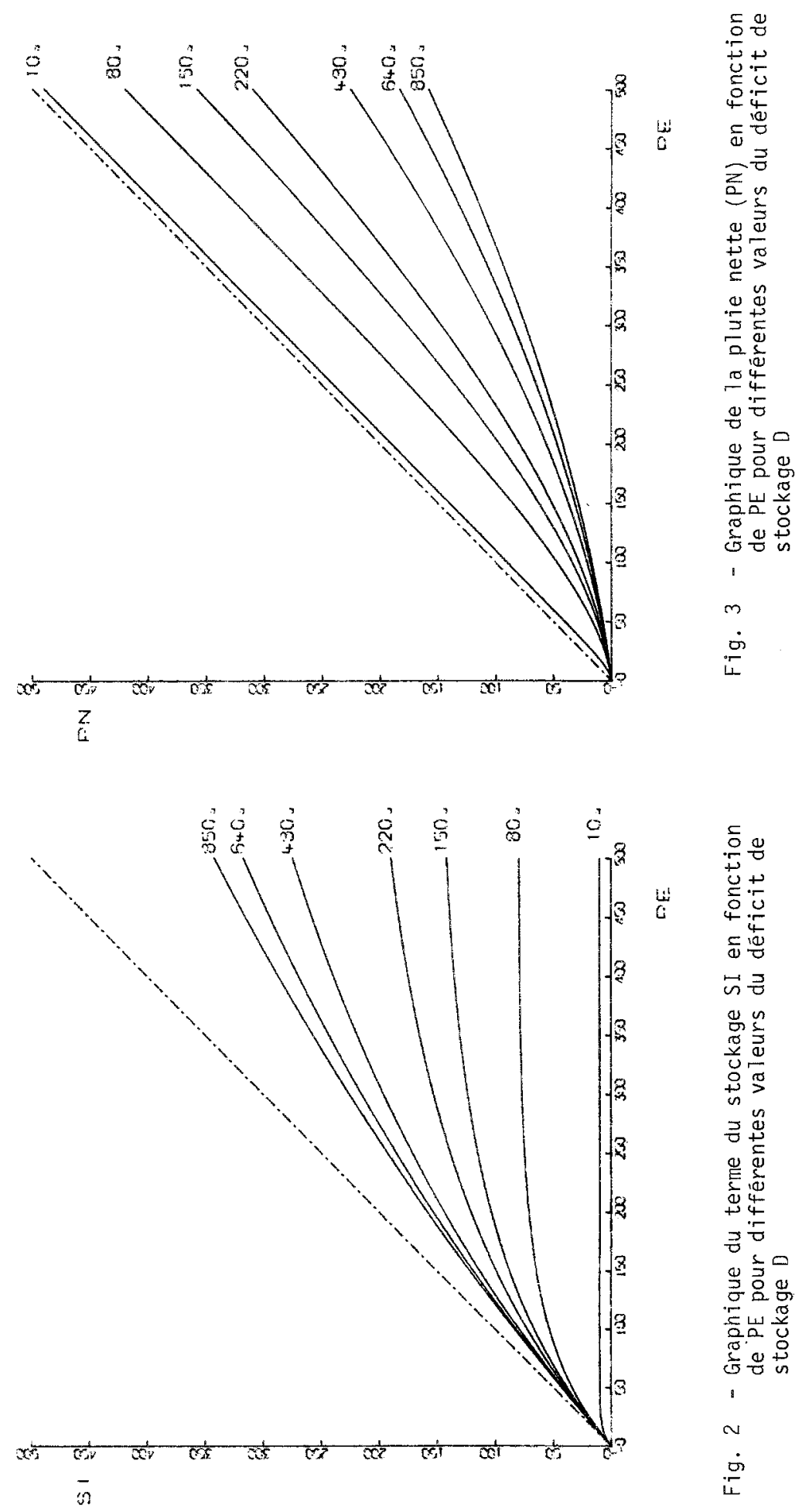


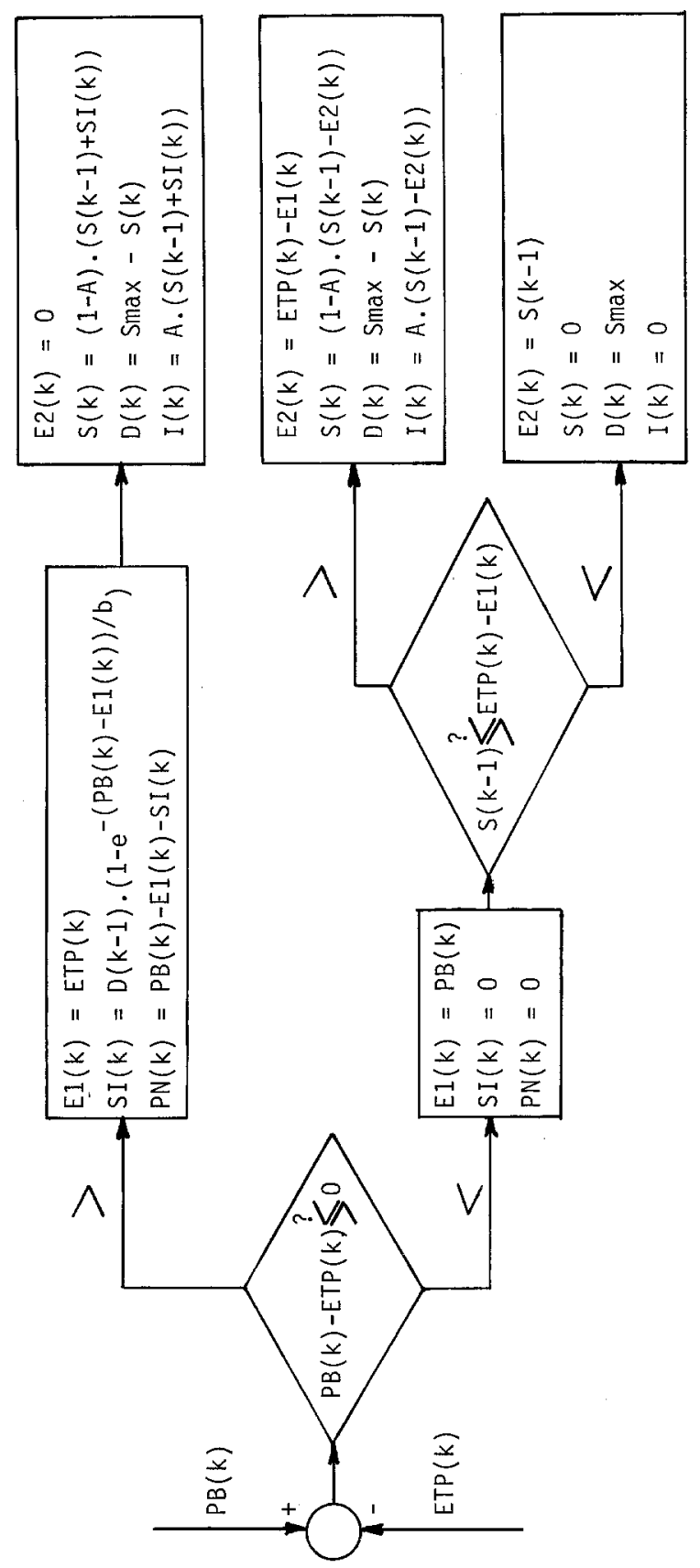

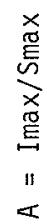

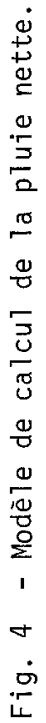

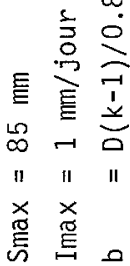

․․․ 


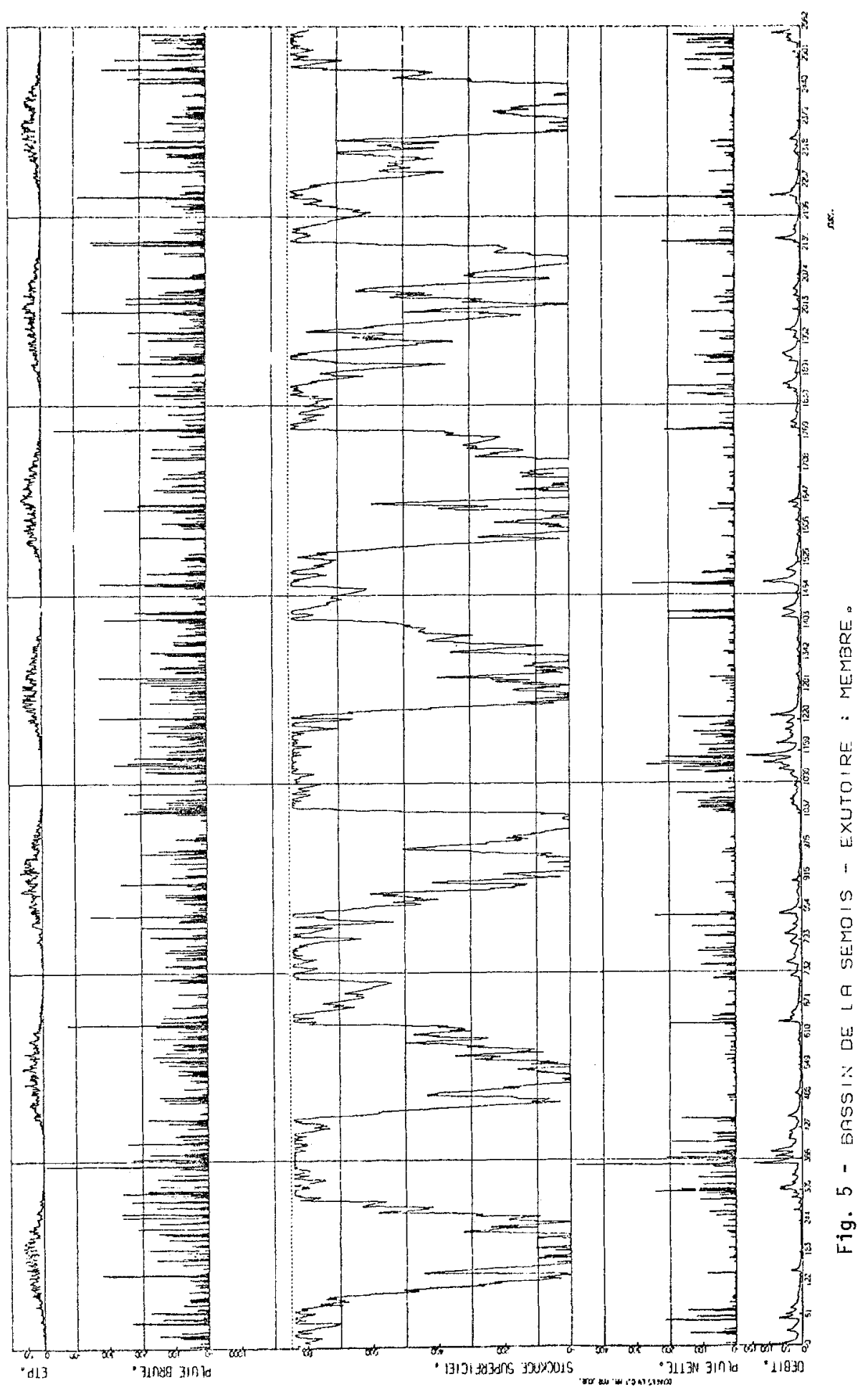


IV. MODELISATION DU DEBIT DE BASE.

IV.1. Lorsque le cours d'eau n'est plus al imentê que par les nappes, la décroissance du débit est décrite par ce que l'on appelle "la courbe de tarissement de la rivière "qui est une caractéristique importante de celle-ci.

Classiquement, la courbe de tarissement sera approximée par l'une des deux relations:

$$
\begin{aligned}
& Q(t) \cong Q_{0} \cdot e^{-\left(t-t_{0}\right) / T H} \\
& Q(t) \cong \frac{Q_{0}}{\left(1+a \cdot\left(t-t_{0}\right)\right)^{2}}
\end{aligned}
$$

oũ : $Q_{0}$ est la valeur du débit à l'instant $t_{0}$ du début du tarissement.

$$
[3],[4],[5] \text {. }
$$

IV.2. L'étude des périodes d'étiage de la Semois nous a permis de constater qu'effectivement la courbe de tarissement peut être bien approximée par une exponentielle décroissance à condition de faire intervenir un terme constant caractêristique de 1 'année considérée. On aura ainsi à la place de (10), la relation:

$$
Q(t)-B_{0} \cong\left(Q_{0}-B_{0}\right) \cdot e^{-\left(t-t_{0}\right) / T H}
$$

oũ $B_{0}$ varie lentement d'une période à l'autre.

11 nous a paru dès lors logique de modéliser le débit de base $B(t)$ en considérant qu'il était composé de deux termes. Ainsi,

$$
B(k)=B R(k)+B L(k)
$$

Le terme $B R(k)$ est un terme qui évolue relativement vite et qui peut être attribué à des nappes, qui, situées près de la surface du sol, subiraient rapidement les effets de recharge et de décharge. Par contre, BL(k) est un terme qui peut se modifier lentement d'année en année et qui serait du à des nappes beaucoup plus stables. Remarque : Dans le cas de la Semois, le terme rapide du débit de base décroit avec une constance de temps TH d'environ 15 jours. Celle-ci est bien différente de la constante de temps des décrues qui est de 1 'ordre de 4 ou 5 jours. 
IV.3. Pour réaliser une bonne simulation du débit d'une rivière, il importe d'avoir une estimation plausible du débit de base tout au long de T'année. C'est à nouveau un problème délicat, d'abord en raison de l'impossibilité qu'il y a de distinguer a priori le débit de base et le débit de ruissellement lorsque ces deux termes coexistent et ensuite, du fait que la dynamique de l'écoulement souterrain est encore très mal connue. En conséquence, nous opterons pour un modèle très rudimentaire mais que nous estimons efficace.

IV.4. Nous modéliserons les termes $B R(k)$ et $B L(k)$ suivant le même schēma. Considérons tout d'abord $B R(k)$.

En période de tarissement, nous savons que $B R(k)$ décroit quasiment comme une exponentielle. Dès lors, l'estimée $\widetilde{B R}(k)$ répondra à la relation :

$$
\widetilde{B R}(k)=x \cdot \widetilde{B R}(k-1)
$$

avec

$$
\alpha=e^{-1 / T H}
$$

oú TH est la constante de temps de la décroissance exponentielle.

Pour décrire la dynamique de $B R(k)$ en période de recharge des nappes, nous utiliserons le volume d'eau $\mathrm{VR}(k)$ stockée par l'ensemble des nappes à réponse rapide :

$$
V R(k)=\sum_{j=k_{0}+1}^{k}[I R(j)-\widetilde{B R}(j)]+V R\left(k_{0}\right)
$$

Dans cette expression, IR(j) représente le terme d'alimentation de ces nappes par infiltration. Nous supposerons que :

$$
\begin{aligned}
\widetilde{B R}(k) & =n \cdot V R(k) \\
\text { En consêquence : } & \\
\widetilde{B R}(k) & =\alpha \cdot \widetilde{B R}(k-1)+(1-\alpha) \cdot I R(k)
\end{aligned}
$$

avec: $\quad \alpha=\frac{1}{1+n}$ 
Lorsque $I R(k)$ s'annule, nous retrouvons ainsi la relation (14).

En utilisant 1 'opérateur de retard $Z^{-1}$, on écrira :

$$
\widetilde{B R}(k)=\frac{1-\alpha}{1-\alpha z^{-1}} \cdot \operatorname{IR}(k)
$$

En procédant de 1a même manière pour $B L(k)$, nous aurons :

$$
\widetilde{B L}(k)=\frac{1-\beta}{1-\beta Z^{-1}} \cdot I L(k)
$$

où cette fois, $\beta$ correspondra à une constante de temps beaucoup plus grande.

Il reste à préciser les termes $I R(k)$ et $I L(k)$.

Nous allons simplement supposer que ce sont des fractions du terme d'infiltration $I(k)$ évoqué dans le paragraphe III moyennant l'utilisation d'un terme de retard correspondant au temps que l'eau met à gagner la nappe. Dẽs lors :

$$
\begin{aligned}
& I R(k)=p \cdot I(k-d r) \\
& I L(k)=q \cdot I(k-d l)
\end{aligned}
$$

Les différents paramētres $\alpha, p, d r, \beta, q$, dl de ce modèle sont optimalisês en utilisant des relevés de débit en période sèche et en minimisant 1 'erreur quadratique moyenne entre le débit de base calculé et observê. Nous avons ainsi retenu les valeurs:

$$
\begin{array}{rlrl}
p & =0.30 & q & =0.69 \\
d r & =5 \text { jours } & d l & =30 \text { jours } \\
\alpha & \rightarrow 15 \text { jours } & \beta & >244 \text { jours }
\end{array}
$$

On Observera que la somme de $p$ et q est voisine de 1 'unité.

Le bilan infiltration - débit de base est ainsi respecté.

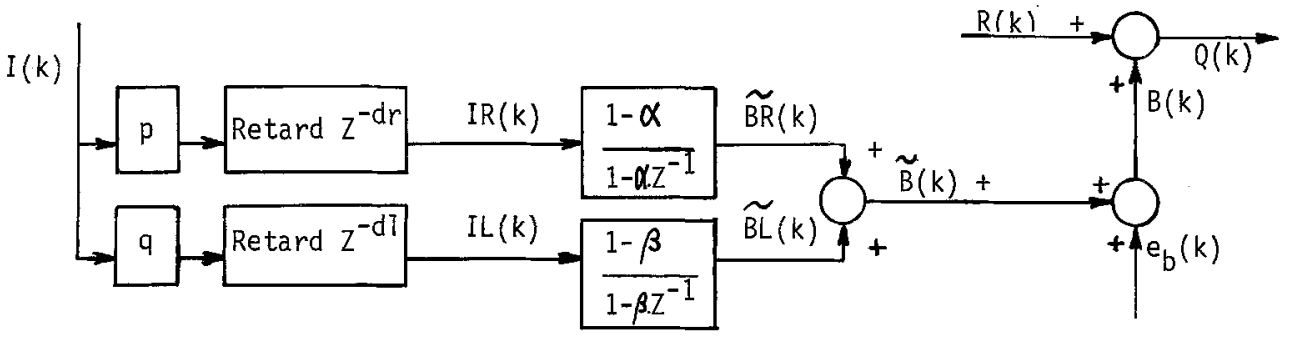

Fig. 6 - Modèle du débit de base 


\section{v. LE MODELE PLUIE NETTE - DEBIT dE RUISSELLEMENT.}

V.1. L'expérience a montré que des modēles 7 inêaires entre la pluie nette et le dēbit de ruissellement donnaient de très bons résultats. Ainsi s'explique le succès de la méthode de 1 'nydrogramme unitaire. [3], [4], [5]

Nous modéliserons le débit de ruisseliement $R(k)$ par l'expression:

$$
\widetilde{R}(k)=\sum_{i=1}^{N}\left[\sum_{j=0}^{M} H(i, j) . P N(i, k-j)\right]
$$

où : PN(i,k) est la pluie nette de la station $i$ pour l'intervalle de temps $k$

$H(i, k)$ est la valeur au temps $k$ de $l$ 'hydrogramme instantané du sous-bassin correspondant â la station $i$

L'expression (24) a toutefois 1 'inconvênient de contenir un très

grand nombre de termes: dans le cas de la Semois, un hydrogramme ne s'annule qu'après 25 ou 30 jours. Il est dès lors plus intéressant d'utiliser l'équation aux différences êquivalente:

$$
\tilde{R}(k)=\sum_{i=1}^{N}\left[\sum_{j=1}^{S} \underset{j}{a} \underset{j}{i}(i, k-j)+\sum_{j=0}^{T} \underset{j}{i} \operatorname{PN}(i, k-j)\right]
$$

ou T'approximation :

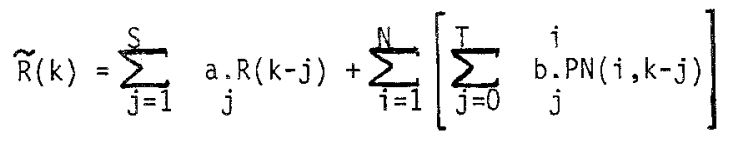

En utilisant $l^{\prime}$ 'opérateur de retard $Z^{-1}$, on écrira plus facilement :

$$
\tilde{R}(k)=A^{\prime}\left(Z^{-1}\right) \cdot R(k-1)+\sum_{i=1}^{N} B^{i}\left(Z^{-1}\right) \cdot P N(i, k)
$$

où $A^{\prime}\left(Z^{-1}\right)=a_{1}+a_{2} Z^{-1}+a_{3} Z^{-2}+\cdots$

$$
8^{i}\left(z^{-1}\right)=b_{0}^{i}+b_{1}^{i} z^{-1}+b_{2}^{i} z^{-2}+\cdots
$$

Si l'on n'utilise que la pluie moyenne sur le bassin, on emploiera le modèle plus simple: 


$$
\tilde{R}(k)=A^{\prime}\left(Z^{-1}\right) \cdot R(k-1)+B\left(Z^{-1}\right) \cdot \overline{P N}(k)
$$

Si $\xi(k)$ est l'erreur du modèle :

$$
\xi(k)=R(k)-\tilde{R}(k)
$$

et en posant : $A\left(Z^{-1}\right)=1-A^{\prime}\left(Z^{-1}\right) \cdot Z^{-1}$

le modèle s'écrira:

$$
\begin{aligned}
& A\left(Z^{-1}\right), R(k)=B\left(Z^{-1}\right) \cdot \overline{P N}(k)+\xi(k) \\
& \text { Le rapport } \frac{B\left(Z^{-1}\right)}{A\left(Z^{-1}\right)} \text { est la fonction de transfert du système et, par }
\end{aligned}
$$

division longue, on peut retrouver la réponse impulsionnelle du système $P N-R$ laquelle est encore appelée hydrogramme instantané dans le contexte hydrologique présent.

\section{La figure 6 montre}

1 'hydrogramme instantané caractēristique de la Semois : ce graphique représente le débit de ruissellement qui serait engendré par une pluie nette de valeur unité qui surviendrait au temps zéro.

Fig. 6

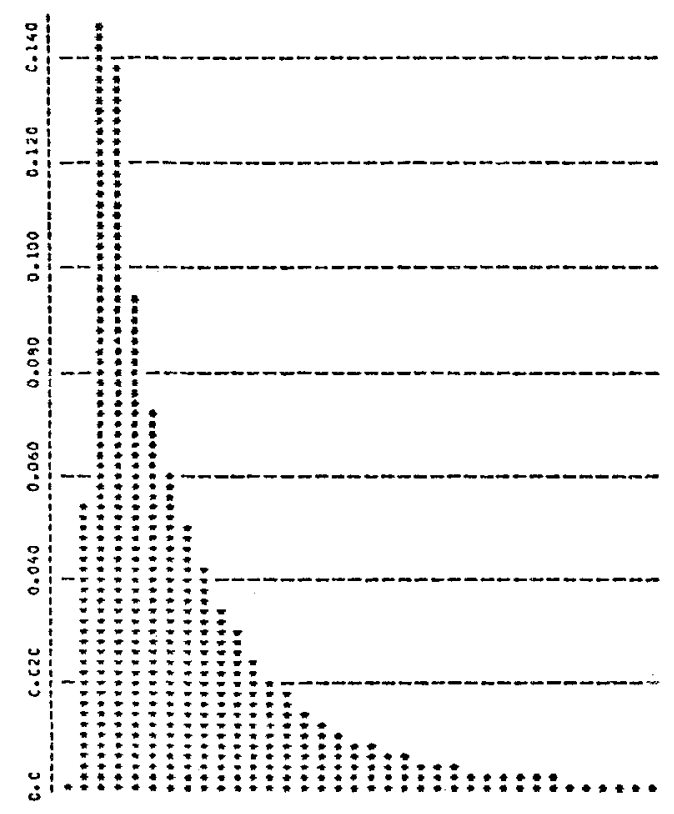

V.2. Pour identifier les paramètres du modèle décrit par la relation (28), nous devons d'abord connaître $R(k)$. Pour ce faire, nous pourrons utiliser l'estimée de $R(k)$ obtenue par la relation :

$$
\hat{R}(k)=Q(k)-\tilde{B}(k)
$$


où $Q(k)$ est le débit total mesuré et $\widetilde{B}(k)$ le débit de base estimé par le sous-modèle du dēbit de base.

L'erreur $\xi(k)$ a actuellement êté modēlisê par l'expression :

$$
\xi(k)=c\left(z^{-1}\right) \cdot e(k)+c
$$

oũ $C\left(Z^{-1}\right)=1+c_{1} \cdot z^{-1}+c_{2} \cdot Z^{-2}+\ldots$

$e(k)$ est supposê être un bruit blanc discret non corrêlé avec l'entrêe PN du modèle.

Le modèle est ainsi décrit par le schéma :

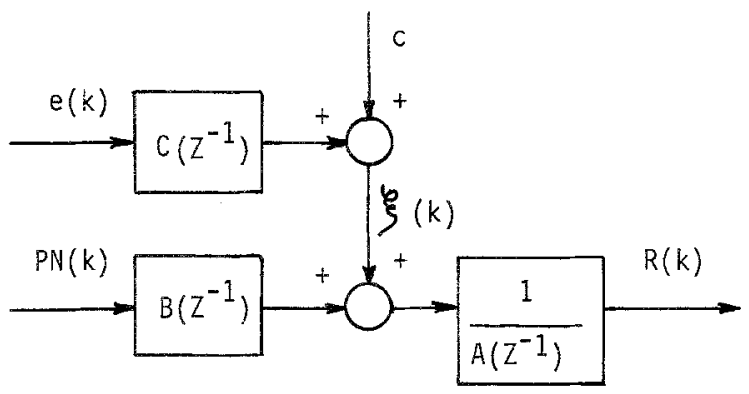

La méthode d'estimation des diffërents paramētres est explicitée dans [8]. Celle-ci est rëcursive et basée sur la minimisation de l'erreur quadratique moyenne $\left(\frac{1}{k}_{j=1}^{k} e^{2}(j)\right)$. On lira également avec intērêt $[10]$.

\section{APPLICATIONS ET RESULTATS SIGNIFICATIFS.}

VI.1. Le modèle que nous avons dêcrit peut tout d'abord servir à la prédiction. Connaissant les pluies et les débits jusqu'aujourd'hui, quel sera le débit le plus probable demain? Nous avons accordé un intérêt tout particulier à cette question et ci-dessous, le lecteur trouvera quelques résultats significatifs.

L'identification du modèie de prédiction à un jour s'est opérée avec un volume de données correspondant à sept années dont avaient été exclues les périodes de neige. Il y avait ainsi 2066 jours de données disponibles.

Bien que, ni les débits, ni la pluie ne soient des processus gaussiens, nous nous contenterons cependant de les caractériser par le contenu du tableau ci-dessous : 


\begin{tabular}{|l|c|c|}
\hline & DEBIT & PLUIE \\
\hline Moyenne (0.1 mm/jour) & 11.7 & 27.9 \\
Variance & 159.4 & 2740 \\
Ecart-type & 12.6 & 52.3 \\
\hline
\end{tabular}

Le tableau suivant reprend les performances de plusieurs modèles élémentaires de prévision à 1 jour en regard de celles du modèle global prêsenté dans cet article. les diffërents modèles sont comparès au moyen de la variance de 1 'erreur de prédiction $\left(\sigma_{\mathrm{e}}^{2}\right)$, de 1 'écart-type de cette erreur $\left(\sigma_{\mathrm{e}}\right)$ et du coefficient $\ell_{g}$ définit par:

$$
e_{g}=\sqrt{1-\frac{\sigma_{e}^{2}}{\sigma_{Q}^{2}}}
$$

où $\sigma_{Q}^{2}$ est la variance du débit.

\begin{tabular}{|c|c|c|c|}
\hline Prédicteurs du débit $Q(k)$ & $\sigma_{e}^{2}$ & $\sigma_{e}$ & eg \\
\hline$\hat{Q}(k / k-1)=a \cdot Q(k-1)+c$ & 11.88 & 3.45 & 0.962 \\
\hline $\begin{aligned} \hat{Q}(k / k-1)= & a_{1} \cdot Q(k-1)+a_{2} \cdot Q(k-2)+a_{3} \cdot Q(k-3) \\
& +C_{1} \cdot e(k-1)+C_{2} \cdot e(k-2)+C_{3} \cdot e(k-3)+c\end{aligned}$ & 10.51 & 3.24 & 0.966 \\
\hline $\begin{aligned} \hat{Q}(k / k-1)= & a_{1} Q(k-1)+a_{2} Q(k-2) \\
& +\sum_{i=1}^{4} b_{i} \cdot P B(k-i) \\
& +\sum_{i=1}^{3} c_{i} \cdot e(k-i)+c\end{aligned}$ & 7.03 & 2.65 & 0.978 \\
\hline $\begin{aligned} \hat{Q}(k / k-1)= & \tilde{B}(k / k-1) \\
& +a_{1} \cdot \hat{R}(k-1)+a_{2} \cdot \hat{R}(k-2) \\
& +\sum_{i=1}^{4} b_{i} \cdot P N(k-i) \\
& +\sum_{i=1}^{3} c_{i} \cdot e(k-i)+c\end{aligned}$ & 3.63 & 1.91 & 0.989 \\
\hline
\end{tabular}

On observera 1 'efficacitê du modèle prêsenté ici.

La référence $[10]$ décrit clairement comment concevoir des prédicteurs à plus long terme. 
VI.2. Ce modêle global peut également étre utilisé pour de la simulation et par exemple pour :

- Préciser le débit possible dans les jours qui viennent à partir des différentes conditions météorologiques probables. Il suffira simplement pour les temps $k$ futurs de remplacer dans les modèles les termes $e(k)$ par 0 et $Q(k)$ par les valeurs calculées et d'introduire pour les entrées pluie et évapotranspiration potentielle les valeurs probables (fig. 7).

- Simuler le débit à partir des relevés de pluies lorsque l'on est en présence de mesures manquantes du débit. A nouveau on remplacera dans le modèle les termes $e(k)$ par 0 et le débit $Q(k)$ par les valeurs calculées

un article en préparation développera en détail ces applications possibles.

Fig. 7

Simulation (trait discontinu) du débit de la Semois en supposant connues les pluies jusqu'à la veille et les débits 5 jours auparavant.

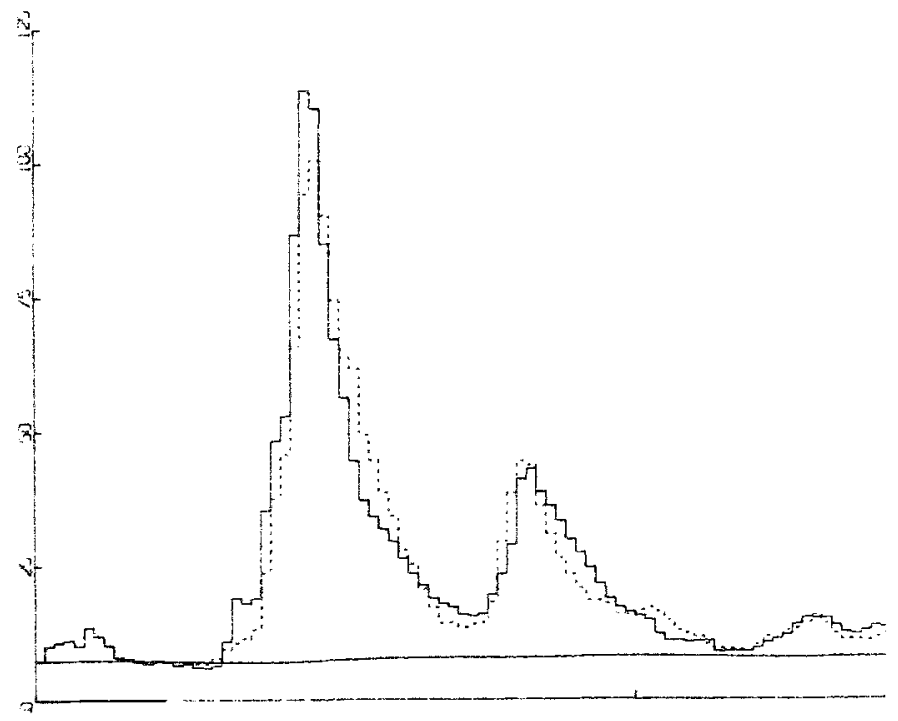

CONCLUSION : Nous avons présentê dans cet article la structure d'un modèle efficace de relation pluie-débit. Il pourra certes être amélioré et un des problèmes délicats à étudier sera celui de la fonte des neiges, sujet qui n'a pas étē abordé ici.

Il importe en tout cas de rappeler que les modèles dynamiques en hydrologie constituent un outil précieux, pour tout qui, à partir d'une meilleure connaissance des phénomènes, souhaite mettre en oeuvre une politique cohërente de gestion des ressources en eau d'un bassin. Et à ce titre, des modèles capables de quantifier l'incidence des pluies sur les débits et de prévoir en conséquence l'évolution possible de ceux-ci, s'avèrent être très utiles. 


\section{REFERENCES :}

[1] BULTOT, F. et DUPRIEZ, G.L. - Estimation des valeurs journalières de l'évapotranspiration potentielle d'un bassin hydrologique. J1 of Hydrology, vol. 21, Ams terdam (1974).

[2] BULTOT, F. et DUPRIEZ, G.L. - Etude hydrométéorologique des précipitations sur les bassins hydrographiques belges. I. Bassin de la Semois. Institut Royal Météorologique de Belgique, Publications, série $A, n^{\circ} 64$.

[3] LARRAS, J. - Prévision et prédétermination des étiages et des crues. Eyrolles, Paris (1972).

[4] ROCHE, M. - Hydrologie de surface. Gauthier-Villars, Paris (1963).

[5] REMENERIAS - L'hydrologie de l'ingénieur. Eyrolles, Paris (1972).

[6] BULTOT, F., DUPRIEZ, G.L. et BODEUX, A. - Interception de la pluie par la végétation forestière. Estimation de l'interception journaZière à Z'aide d'un modèle mathématique. Jl of Hydrology, 17, (1972), 193-223.

[7] de MARSILY, G. - La relation pluie-débit dons le bassin versant de L'HaZZue. Ecole Nationale des Mines de Paris, Centre d'Informatique Géologique (77 - Fontainebleau, 35 rue Saint-Honoré) (1971).

[8] KASHYAP, R.L. et RAO, A.R. - Real time recursive prediction of miver flows. Automatica 9 (1973), 175-183.

[9] CORLIER, F. - Modèle mathématique des débits joumaliers de la Sambre. Faculté des Sciences Agronomiques de Gembloux, Belgique (1974).

[10] JENKINS, G.M. et BOX, G.E.P. - Time series conalysis forecasting and control. Holden Day (1970). 